\title{
CRITÉRIOS PARA A CERTIFICAÇÃO DE PRODUTOS NA REGULAMENTAÇÃO DO INMETRO
}

\author{
Roberta de Freitas Chamusca
}

Danielle Assafin Vieira Souza Silva

Cristiane Mascarenhas da Silva Sampaio

Raphael Carlos Santos Machado

\begin{abstract}
Resumo
O Instituto Nacional de Metrologia, Qualidade e Tecnologia (Inmetro) utiliza, sistematicamente, a avaliação da conformidade em sua regulamentação. Na certificação de produtos, processos e serviços, há sete tipos de esquemas possíveis. No entanto, na inexistência de regras explícitas, observa-se, nos programas instituídos pelo Inmetro, a preferência pelo tipo $1 \mathrm{~b}$, ou certificação de lote, e pelo tipo 5, o mais completo dos esquemas, no que se refere às atividades de avaliação empregadas. A partir da revisão bibliográfica, este trabalho investigou como selecionar o esquema adequado na certificação. Foi elaborada uma sistemática de apoio à decisão, baseando-se nas variáveis relacionadas ao produto, processo produtivo, setor produtivo, mercado e canal de distribuição. A sistemática potencialmente promove a melhoria da qualidade regulatória do Inmetro e o aumento da transparência, além de constituir-se ferramenta útil para outros atores, do setor público ou privado, que sejam proprietários de esquemas de certificação.
\end{abstract}

Palavras-chave: Regulamentação. Avaliação da conformidade. Certificação de produtos. Esquemas de certificação. Inmetro.

\section{CRITERIA FOR PRODUCT CERTIFICATION IN INMETRO REGULATION ABSTRACT}

\begin{abstract}
The Brazilian Institute of Metrology, Quality and Technology (Inmetro) systematically uses conformity assessment in its regulation. In product, process, and service certification, there are seven types of possible schemes. However, in the absence of explicit rules in Inmetro, there is a preference for type $1 \mathrm{~b}$, or batch certification, and for type 5, which is the most complete scheme regarding the evaluation activities that are employed. Based on a literature review, this work investigated how to select the appropriate scheme in certification. A decision systematic was elaborated, based on the variables related to the product, production process, productive sector, market and distribution channel. The framework potentially promotes the improvement of Inmetro's regulatory quality and increases transparency, besides being a useful tool for other players, both in public or private sector, who act as a certification scheme owner.
\end{abstract}

Keywords: Regulation. Conformity assessment. Product certification. Certification schemes. Inmetro. 


\section{INTRODUÇÃO}

O Inmetro foi criado a partir da Lei no 5.966/1973 e, desde o início da década de 80, logo após a instituição do extinto Sistema Brasileiro de Certificação (atual Sistema Brasileiro de Avaliação da Conformidade - SBAC), passou a estabelecer programas de avaliação da conformidade (PAC). Até 2011, o Inmetro dependia da formalização do Conmetro sobre as áreas que deveria ou poderia regulamentar (BRASIL, 1999). A Lei n ${ }^{\circ}$ 2.545/2011 explicitou a competência do Inmetro para elaborar e expedir regulamentos técnicos no campo da avaliação da conformidade de produtos, processos e serviços, nas áreas que não constituam objeto da competência de outros órgãos ou entidades da administração pública federal, no que se refere a aspectos relacionados com segurança, prevenção de práticas enganosas de comércio, proteção da vida e saúde humana, animal e vegetal, e meio ambiente (BRASIL, 2011).

No cumprimento da sua atividade de regulamentação, o Inmetro vem fazendo uso, sistematicamente, da avaliação da conformidade para demonstrar o atendimento aos requisitos técnicos estabelecidos. Os PAC estabelecidos pelo Inmetro, no âmbito do atual Sistema Brasileiro de Avaliação da Conformidade (SBAC), compreendem um conjunto de documentos que definem os requisitos a serem atendidos por um objeto e o passo a passo para sua avaliação da conformidade, utilizando-se de diferentes mecanismos para a atestação da conformidade, tais como a certificação, a declaração da conformidade do fornecedor (DF) e a inspeção. Outros regulamentadores, como a Agência Nacional de Vigilância Sanitária (Anvisa), o Conselho Nacional de Trânsito (Contran), o Conselho Nacional do Meio Ambiente (Conama), o Ministério da Agricultura e o recém extinto Ministério do Trabalho, dentre outros, também têm se apoiado em programas estabelecidos pelo Inmetro para verificar o atendimento aos requisitos de seus regulamentos expedidos.

A partir de dados extraídos do sítio do Inmetro, em fevereiro de 2019, contabilizamos 185 PAC publicados pelo Inmetro, seja por iniciativa própria ou por delegação de competência de outros regulamentadores. Desse total, 140 adotam a certificação como forma de atestar a conformidade de produtos, processos, serviços, pessoas ou sistemas de gestão, o que corresponde a aproximadamente $76 \%$ dos PAC publicados. O histograma apresentado na Figura 1 mostra a evolução dos programas publicados entre 2002 e 2018, evidenciando o predomínio da certificação sobre os demais mecanismos de avaliação da conformidade. 
Figura 1: PAC publicados entre 2002 e 2018, agrupados de acordo com o mecanismo de avaliação da conformidade utilizado. Falar da etiquetagem.

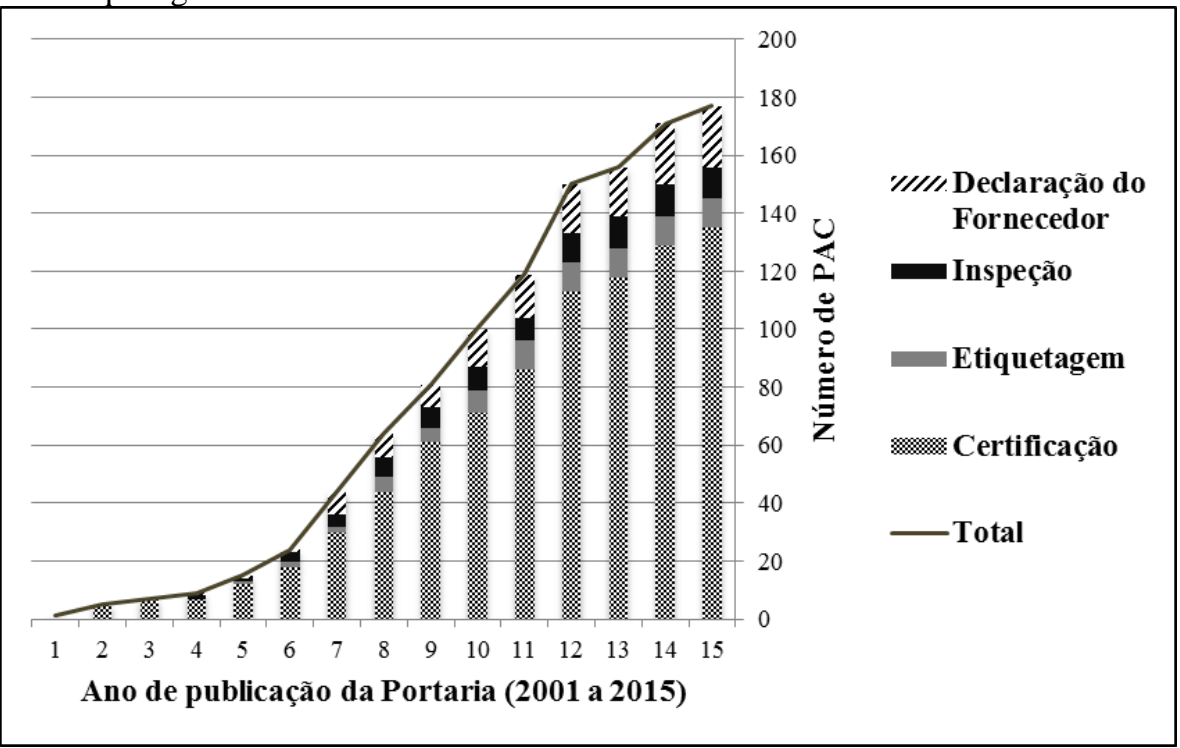

Fonte: Elaboração própria (a partir de INMETRO, 2019).

No caso específico da certificação de produtos (que abrange também serviços e processos), o Inmetro especifica sete modelos de certificação, baseados nos tipos de esquemas de certificação descritos na norma ISO/IEC 17067 (ABNT, 2015), e que se diferenciam essencialmente pela realização ou não de auditoria do Sistema de Gestão da Qualidade (SGQ) do fabricante/prestador de serviço, pela existência ou não da Avaliação de Manutenção (repetição periódica das atividades de avaliação da conformidade para manutenção da certificação) e pelo tipo de atividade de avaliação da conformidade realizada na Avaliação de Manutenção.

O Guia de Boas Práticas de Regulamentação (CONMETRO, 2007), preconiza que os procedimentos de avaliação da conformidade adotados devem assegurar o mais elevado grau de conformidade com a menor intervenção governamental possível e com os menores custos para os regulamentadores e regulamentados. Não obstante essa diretriz, observa-se o predomínio da aplicação do modelo de certificação 5, que pode ser considerado um dos mais rigorosos em função das atividades de avaliação da conformidade que emprega (ensaios e/ou inspeções, avaliação do processo produtivo e auditoria do SGQ).

A partir do levantamento das informações contidas nos programas de certificação de produtos, processos e serviços publicados pelo Inmetro no período de 2001 a 2015, Chamusca, Sampaio e Silva (2015), constataram que o modelo de certificação 5 é o mais adotado, tendo sido referenciado em 110 dos 126 programas, o que corresponde a uma frequência de utilização 
de $87 \%$. O segundo modelo mais utilizado é o 7 (atual 1b), referenciado em 65 RAC, o que corresponde a $52 \%$ dos programas de certificação de produtos, processos e serviços.

Se por um lado, a preponderância do modelo de certificação 5 sobre os demais modelos pode ser vista como uma atitude conservadora e de fortalecimento da atividade de avaliação da conformidade enquanto avaliação pré-mercado, por outro lado, corre-se o risco de criar custos desnecessários para o setor regulado e, em última instância, para o consumidor final. A ausência do estabelecimento de regras para a seleção dos modelos de certificação de produtos confere subjetividade ao processo de desenvolvimento de PAC e compromete a harmonização nas práticas de avaliação da conformidade.

A questão que se apresenta é como realizar a seleção de um ou mais tipos de esquemas para a certificação de um dado produto, processo ou serviço? A contribuição deste trabalho reside no fato de não apenas indicar as variáveis que influenciam a seleção dos tipos de esquemas de certificação, o que já se acha na literatura, mas de reunir um conjunto de variáveis e propor uma correlação com as características dos tipos de esquemas de certificação, de tal maneira que seja possível definir qual tipo de esquema utilizar em função dos valores assumidos pelas variáveis.

A seguir, na segunda seção, é apresentada a revisão bibliográfica. Para a seleção das fontes bibliográficas foi adotado o processo sistemático proposto por Russo, Macedo-Soares e Villas (2006). A busca de artigos relacionados ao tema de estudo foi realizada através do Portal Capes. Além de artigos, foram selecionadas dissertações de mestrado e teses de doutorado. As palavras-chave para a seleção dos textos foram utilizadas nos idiomas português e inglês, da seguinte forma: avaliação da conformidade, conformity assessment, esquema de certificação, modelo de certificação, certification scheme, certificação de produto, product certification. A busca com palavras-chave em português gerou um baixo número de textos e, portanto, não foram aplicados filtros adicionais. Já para o inglês, optou-se por utilizar um filtro temporal de artigos publicados nos últimos 10 anos, além de um refinamento da busca por tópicos afins ao tema de pesquisa, possibilitando a seleção total de nove artigos de interesse.

Esse reduzido número de publicações já era esperado, já que o tema abordado é ainda explorado com timidez na área acadêmica. Sendo assim, recorreu-se também aos principais fóruns que discutem a avaliação da conformidade, destacando-se o Comitê de Avaliação da Conformidade da Organização Internacional para Normalização (ISO/CASCO) em âmbito internacional e o Inmetro no plano nacional. As normas técnicas do ISO/CASCO, cuja maioria é internalizada no Brasil pela Associação Brasileira de Normas Técnicas (ABNT), também compuseram a base da pesquisa bibliográfica. A partir da aplicação das palavras-chave já 
referidas, selecionou-se inicialmente 14 documentos. A revisão bibliográfica incluiu outras referências, geralmente citadas nas obras primeiramente selecionadas, com destaque às publicações da Cooperação Econômica Ásia-Pacífico (APEC), da Comissão Europeia e da Comissão Econômica das Nações Unidas para a Europa (UNECE).

A terceira seção dedica-se à descrição do método desta pesquisa. Em sequência, na quarta parte do artigo, é apresentada a sistemática para a seleção dos tipos de esquemas de certificação. A quinta seção, finalmente, traz as considerações finais, com conclusões sobre as proposições de pesquisa e os desafios e possibilidades de aplicação da sistemática.

\section{REFERENCIAL TEÓRICO}

\subsection{AVALIAÇÃO DA CONFORMIDADE E CERTIFICAÇÃO DE PRODUTOS}

A avaliação da conformidade é definida como a "demonstração de que os requisitos especificados relativos a um produto, processo, sistema, pessoa ou organismo são atendidos." (ABNT, 2005b, p. 1). Os sistemas e esquemas de avaliação da conformidade devem ser estabelecidos segundo o princípio da abordagem funcional definido pela ISO. Por ele, as seguintes funções são englobadas: Seleção, Determinação, Análise, Atestação e, quando aplicável, Supervisão. Quanto às formas de atestação, há a de primeira parte, ou declaração do fornecedor, quando o próprio fornecedor do produto declara a conformidade, ou de terceira parte, ou certificação, quando a atestação é realizada por um organismo independente do fornecedor do objeto de avaliação da conformidade (ABNT, 2005b, 2015).

A certificação de produtos, processos e serviços recebe o termo genérico de “certificação de produtos" (ABNT, 2015) e é conduzida por organismos de certificação de produtos (OCP). É uma atividade que abrange países desenvolvidos e em desenvolvimento e tem uma história muito mais longa do que a certificação de sistemas de gestão (ISO; UNIDO, 2010).

Em 1980, a ISO publicou um manual sobre os princípios e práticas de certificação, motivada pela escassez de documentação existente sobre o tema na época. Considerando que diversos países já realizavam certificações, mas de formas às vezes distintas, a ISO percebeu a necessidade de harmonizar as práticas, sendo possível uma aceitação mútua dos sistemas de certificação, eliminando barreiras desnecessárias ao comércio entre países (ISO, 1982). O manual da ISO estabeleceu oito sistemas de certificação, com diferentes graus de confiança de que os produtos atendiam a requisitos especificados, que foram desenvolvidos para serem 
aplicados em circunstâncias variadas, como, por exemplo, para a certificação de diferentes tipos de produtos, técnicas de fabricação ou necessidades de compradores (ISO, 1982).

Em 1992, a ISO fez um apanhado dos guias publicados pela organização na década anterior e promoveu alterações nos sistemas de certificação. Uma delas foi a introdução do conceito de supervisão, que tem como objetivo verificar se os produtos fabricados após a certificação inicial continuam atendendo aos requisitos. Outra mudança se refere aos ensaios de verificação nos sistemas de certificação 4 e 5 para conferir a continuidade da conformidade da produção. Nas definições de 1980, os ensaios eram feitos a partir da coleta de amostras na fábrica e no comércio ao mesmo tempo. Em função dos elevados custos associados à atividade, os ensaios passaram, então, a ser realizados em amostras coletadas na fábrica e/ou no comércio, sendo possível alternar os locais de coleta das amostras (ISO, 1992).

Somente no ano de 2004, a ISO publicou um guia para a certificação de produtos, o ISO/IEC Guia 67, que sistematizou ainda mais o processo de certificação de produtos, não apenas definindo os sistemas de certificação, mas também introduzindo as etapas funcionais básicas que deveriam compor todos os esquemas de certificação. Em 2013, o Guia 67 deu lugar à norma ISO/IEC 17067, cuja internalização pela ABNT foi concluída em 2015. A norma introduziu diretrizes para o estabelecimento e operação de esquemas de certificação de produtos e enfatizou a abordagem funcional para a certificação, segmentando-a nas funções de seleção, determinação, análise crítica, decisão e atestação (ABNT, 2015).

Os tipos de esquemas de certificação de produtos estabelecidos pela norma ISO/IEC 17067 estão resumidos no Quadro 1 a seguir.

Quadro 1. Tipos de esquemas de certificação de produtos estabelecidos pela ISO.

\begin{tabular}{|c|l|}
\hline $\begin{array}{c}\text { Tipo de } \\
\text { esquema }\end{array}$ & \multicolumn{1}{c|}{ Características } \\
\hline $\mathbf{1 a}$ & $\begin{array}{l}\text { Uma ou mais amostras do produto são submetidas às atividades de determinação. Um Certificado } \\
\text { de Conformidade é emitido para o tipo de produto. Itens de produção subsequentes não são } \\
\text { abrangidos pela atestação do OCP. }\end{array}$ \\
\hline $\mathbf{1 b}$ & $\begin{array}{l}\text { Envolve a certificação de todo um lote de produtos. A proporção a ser ensaiada pode incluir } \\
\text { ensaio de todas as unidades do lote ou de parte dele. Em caso de conformidade, todos os itens do } \\
\text { lote estão certificados e podem ter uma marca de conformidade afixada. }\end{array}$ \\
\hline $\mathbf{2}$ & $\begin{array}{l}\text { A parte da supervisão envolve a retirada periódica de amostras do produto do mercado. Esse } \\
\text { esquema pode identificar o impacto do canal de distribuição sobre a conformidade, mas os } \\
\text { recursos que ele requer podem ser extensos. Quando não conformidades significativas forem } \\
\text { encontradas, as medidas corretivas podem ser limitadas, uma vez que o produto já foi distribuído } \\
\text { para o mercado. }\end{array}$ \\
\hline
\end{tabular}




\begin{tabular}{|c|l|}
\hline $\begin{array}{c}\text { Tipo de } \\
\text { esquema }\end{array}$ & \multicolumn{1}{c|}{ Características } \\
\hline $\mathbf{3}$ & $\begin{array}{l}\text { A supervisão envolve a retirada periódica de amostras do produto do ponto de produção e inclui } \\
\text { a avaliação periódica do processo de produção. Não fornece qualquer indicação do impacto que } \\
\text { o canal de distribuição desempenha sobre a conformidade. Quando graves não conformidades } \\
\text { forem encontradas, pode haver oportunidade de resolvê-las antes de ocorrer a distribuição } \\
\text { generalizada ao mercado. }\end{array}$ \\
\hline $\mathbf{4}$ & $\begin{array}{l}\text { A supervisão permite a escolha entre a retirada periódica de amostras do produto do ponto de } \\
\text { produção, ou do mercado, ou ambos, e inclui a avaliação periódica do processo de produção. } \\
\text { Pode indicar o impacto do canal de distribuição sobre a conformidade e fornece um mecanismo } \\
\text { de pré-mercado para identificar e resolver graves não conformidades. Duplicaçães significativas } \\
\text { de esforços podem ocorrer para produtos cuja conformidade não é afetada durante o processo de } \\
\text { distribuição. }\end{array}$ \\
\hline $\mathbf{5}$ & $\begin{array}{l}\text { A supervisão permite a escolha entre a retirada periódica de amostras do produto do ponto de } \\
\text { produção, ou do mercado, ou ambos, e inclui a avaliação periódica do processo de produção ou } \\
\text { auditoria do Sistema de Gestão (SG), ou ambos. A extensão pela qual as quatro atividades de } \\
\text { supervisão são conduzidas pode ser variada para uma determinada situação, conforme definido } \\
\text { no esquema. Se a supervisão incluir a auditoria do SG, uma auditoria inicial do SG será } \\
\text { necessária. }\end{array}$ \\
\hline \multirow{6}{*}{$\begin{array}{l}\text { Aplica-se, principalmente, à certificação de processos e serviços. Em algumas situações, os } \\
\text { elementos tangíveis de um serviço podem suportar a evidência de conformidade. Convém que a } \\
\text { parte de supervisão desse esquema inclua auditorias periódicas do SG e a avaliação periódica do } \\
\text { serviço ou processo. }\end{array}$} \\
\hline
\end{tabular}

Fonte: Elaboração própria (com base em ABNT, 2015).

\subsection{USO DA AVALIAÇÃO DA CONFORMIDADE NA REGULAMENTAÇÃO TÉCNICA}

Segundo a UNECE (2012), a avaliação da conformidade pode ser considerada uma ferramenta de mitigação de riscos para fins regulatórios, na medida em que previne que produtos não conformes sejam colocados no mercado. A avaliação da conformidade inserida em um contexto de regulamentação pode ser considerada, portanto, como uma ferramenta de controle de pré-mercado.

Na medida em que os regulamentadores frequentemente exigem a demonstração de que produtos e processos produtivos atendem aos requisitos mínimos para assegurar a proteção à saúde, à segurança e ao meio ambiente, a avaliação da conformidade surge como uma ferramenta para evidenciar a observância aos requisitos estabelecidos em regulamentos técnicos. No entanto, embora a avaliação da conformidade possa ser útil, ela também pode causar prejuízos se conduzida de forma inapropriada (APEC, 2013). 
Assim, um grande desafio dos regulamentadores reside na escolha entre os procedimentos de avaliação da conformidade, principalmente na opção entre a adoção da avaliação da conformidade de primeira ou de terceira parte. Sob esse aspecto, a Rede para Metrologia, Acreditação e Normalização de Países em Desenvolvimento (DCMAS) recomenda que os procedimentos de avaliação da conformidade sejam escolhidos com base na avaliação dos riscos de um produto ou processo específico (DCMAS, 2010). Portanto, é razoável esperar que os regulamentadores optem pelo uso da DF (avaliação da conformidade de primeira parte) para produtos de baixo risco e adotem a certificação ou inspeção de terceira parte para produtos mais complexos e com elevado grau de risco (UNECE, 2012).

Segundo a ISO ([201-]), alguns aspectos-chave devem ser levados em consideração ao fazer uso da avaliação da conformidade na atividade de regulamentação. Entre eles, incluemse o uso da abordagem funcional no estabelecimento de esquemas de avaliação da conformidade, a consideração sobre os custos e investimentos associados aos procedimentos de avaliação da conformidade e a necessidade de existência de recursos competentes (organismos de avaliação da conformidade e especialistas) para conduzir as atividades de avaliação da conformidade. As boas práticas regulatórias, o risco e as atividades de acompanhamento também são considerados pela ISO fatores-chave para a associação da avaliação da conformidade à regulamentação (ISO, [201-]).

Em análise comparativa entre a União Europeia, países da APEC e o Brasil sobre as estratégias e os fatores que influenciam a escolha do procedimento de avaliação da conformidade, verificou-se que, enquanto o continente europeu adota a declaração do fornecedor como a forma de atestação da conformidade, o Brasil e os países da APEC utilizam atestação tanto de $1^{\mathrm{a}}$ parte (DF) como a de $3^{\mathrm{a}}$ parte. Os três elementos de análise consideram o risco como fator que influi na escolha do procedimento de avaliação da conformidade, bem como o tipo ou as características do produto, sem esclarecer, no entanto, como esses fatores influenciam as decisões sobre as regras da avaliação da conformidade. O Quadro 2 resume a análise comparativa realizada. 
Quadro 2: Fatores que influenciam as estratégias de avaliação da conformidade.

\begin{tabular}{|c|c|c|c|}
\hline \multicolumn{4}{|c|}{$\begin{array}{c}\text { Diretrizes do ISO/CASCO (ISO, [201-]) para uso da avaliação da conformidade na atividade de } \\
\text { regulamentação }\end{array}$} \\
\hline \multicolumn{4}{|c|}{ - Uso da abordagem funcional descrita nas normas de avaliação da conformidade; } \\
\hline \multirow{4}{*}{\multicolumn{4}{|c|}{$\begin{array}{l}\text { - Disponibilidade de expertise para a condução das atividades de avaliação da conformidade; } \\
\text { - Natureza dos riscos que se pretende mitigar com o uso da avaliação da conformidade; } \\
\text { - Complexidade dos procedimentos de avaliação da conformidade, seus benefícios decorrentes e seus custos } \\
\text { associados; }\end{array}$}} \\
\hline & & & \\
\hline & & & \\
\hline & & & \\
\hline \multicolumn{4}{|c|}{ - Condução de atividades de acompanhamento no mercado, que incluem a Supervisão e a Fiscalização. } \\
\hline Região/País & União Europeia & Países da APEC & Brasil (SBAC) \\
\hline $\begin{array}{l}\text { Estratégias de } \\
\text { avaliação da } \\
\text { conformidade }\end{array}$ & $\begin{array}{l}\text { Atestação de } 1^{\text {a }} \text { parte } \\
\text { (DF). }\end{array}$ & $\begin{array}{l}\text { - Sistemas híbridos de } \\
\text { certificação voluntária e } \\
\quad \text { compulsória; } \\
\text { - Sistemas que utilizam tanto a } \\
\text { atestação de } 1^{\mathrm{a}} \text { parte }(\mathrm{DF}) \\
\text { como a de } 3^{\mathrm{a}} \text { parte. }\end{array}$ & $\begin{array}{l}\text { - Certificação; } \\
\text { - Inspeção; } \\
\text { - DF. }\end{array}$ \\
\hline $\begin{array}{c}\text { Fatores que } \\
\text { influenciam a } \\
\text { escolha do } \\
\text { procedimento } \\
\text { de avaliação da } \\
\text { conformidade }\end{array}$ & $\begin{array}{l}\text { - Nível de risco } \\
\text { envolvido e grau de } \\
\text { segurança requerido; } \\
\text { - Tipo de produto; } \\
\text { - Métodos de produção; } \\
\text { - Infraestrutura } \\
\text { econômica do setor } \\
\text { produtivo. }\end{array}$ & $\begin{array}{l}\text { - Risco; } \\
\text { - Setor regulamentado; } \\
\text { - Tipo de produto. }\end{array}$ & $\begin{array}{c}\text { - Características do produto; } \\
\text { - Risco do produto; } \\
\text { - Infraestrutura de avaliação } \\
\text { da conformidade existente; } \\
\text { - Dificuldade de } \\
\text { acompanhamento no } \\
\text { mercado; } \\
\text { - Características do setor } \\
\text { produtivo (ex.: tecnologia, } \\
\text { presença de micro e } \\
\text { pequenas empresas). }\end{array}$ \\
\hline
\end{tabular}

Fonte: Elaboração própria (com base em APEC,2013; Conmetro, 2007; Inmetro, 2015a; ISO [201-]; EU, 2008).

\subsection{METODOLOGIAS EXISTENTES PARA A SELEÇÃO DE ESQUEMAS DE AVALIAÇÃO DA CONFORMIDADE}

$\mathrm{Na}$ investigação de metodologias utilizadas para a seleção de esquemas de avaliação da conformidade, destaca-se o procedimento elaborado para compras públicas de produtos e serviços de tecnologia da informação e comunicação (TIC) acessíveis, desenvolvido pelo Comitê Europeu de Normalização (CEN, 2008), bem como a sistemática aplicável à avaliação da conformidade de objetos que compõem a infraestrutura do setor de gás brasileiro (FOSSA, 2012), que se baseia no método original proposto por CEN (2008). Ambos têm em comum o uso da abordagem funcional da ISO/CASCO. Desta forma, eles decompõem cada função de avaliação da conformidade em uma ou mais "dimensões".

As "dimensões" são associadas a "critérios", que são derivados de "elementos". Enquanto CEN (2008), estabelece critérios para cinco elementos distintos (produto, mercado, usuário, órgão da administração pública responsável pela aquisição do produto e processo de 
compras públicas), Fossa (2012), considera apenas quatro elementos relacionados ao setor de gás, quais sejam o produto, o mercado, o usuário e o comprador do produto. Para cada cenário particular de um objeto de avaliação da conformidade, os "critérios" assumem valores específicos, que influenciam os valores recomendados para as dimensões e impacta na escolha do esquema de avaliação da conformidade a ser adotado.

Há, ainda, a proposta específica para seleção de sistemas de certificação de produtos elaborada por Barbulio Filho (2012). O autor dá ênfase às características do processo produtivo para a seleção dos sistemas de certificação. Essas características abrangem a capabilidade do processo, o seu grau de automação, o seu nível de controle, as ferramentas da qualidade empregadas pelo fabricante no controle do processo e as formas de controle das matériasprimas.

Aliás, a influência do processo produtivo na escolha dos procedimentos de avaliação da conformidade é percebida em várias aplicações nos diversos países. No Canadá, a avaliação da conformidade de terceira parte é adotada em setores que não tem tradição de alta qualidade (APEC, 2013). Para o CONMETRO (2007), pode ser desejável que os fornecedores disponham de sistemas de gestão para assegurar o fornecimento de produtos e serviços em conformidade com os requisitos regulamentares e a velocidade do aperfeiçoamento tecnológico do setor é um fator a ser considerado na escolha dos procedimentos de avaliação da conformidade.

Na União Europeia, a seleção dos módulos de avaliação da conformidade deve considerar os métodos de produção (CE, 2015), abrandando as regras de avaliação da conformidade para produtos artesanais ou fabricados sob encomenda e para pequenas produções em série (UE, 2008). Para a ISO (1982), os sistemas de certificação foram desenvolvidos com o propósito de serem aplicados em circunstâncias variadas, levando-se em consideração as diferentes técnicas de fabricação (ISO, 1982). A proposta de Barbulio Filho (2012), nesse sentido, tem o mérito de explicitar como se dá a influência das características dos processos produtivos na escolha dos procedimentos de avaliação da conformidade, o que não havia sido feito de forma tão evidente nas referências destacadas acima.

Conforme o método de pesquisa apresentado na sessão que se segue, a identificação das variáveis utilizadas na sistemática proposta por este trabalho foi possível graças à revisão bibliográfica e sua estruturação foi também fundamentada na abordagem funcional utilizada nos métodos para seleção de esquemas de avaliação da conformidade propostos por CEN (2008) e Fossa (2012). 


\section{MÉTODO}

A pesquisa foi estruturada em três fases. Primeiramente, na revisão bibliográfica, foram estabelecidos os seguintes três objetivos: i) buscar os conceitos relativos à avaliação da conformidade, certificação de produtos, regulação e regulamentação; ii) identificar estratégias de uso da avaliação da conformidade por diferentes regulamentadores; e iii) identificar métodos existentes para a seleção de esquemas de avaliação da conformidade. Para isso, foi utilizada a sistemática proposta por Russo, Macedo-Soares e Villas (2006), descrita anteriormente.

Já na fase de análise, inicialmente, o método proposto por CEN (2008), para a seleção de esquemas de avaliação da conformidade foi customizado para a seleção dos tipos de esquemas de certificação de produtos a partir da decomposição, em dimensões, das funções de avaliação da conformidade relativas à certificação de produtos e da valoração dessas dimensões. Em seguida, foi feita a identificação das variáveis que influenciam a seleção dos tipos de esquemas, bem como a correlação entre as variáveis e os tipos de esquemas. A identificação das variáveis e sua associação com as dimensões das funções de certificação também é resultado da leitura interpretativa do referencial teórico adotado.

Por fim, a terceira fase da pesquisa se concentrou na estruturação do passo a passo para a seleção dos tipos de esquemas de certificação de produtos, que é o resultado principal deste trabalho.

\section{APRESENTAÇÃO DOS RESULTADOS}

\subsection{VARIÁVEIS QUE INFLUENCIAM A SELEÇÃO DO TIPO DE ESQUEMA DE CERTIFICAÇÃO}

Foram identificadas 12 variáveis que influenciam a seleção do tipo de esquema de certificação, dependentes dos elementos "produto "setor produtivo", "mercado", processo produtivo" e "canal de distribuição", conforme demonstra a 


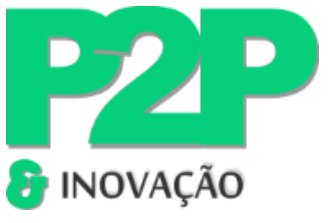

Figura 1. 
Figura 1: Variáveis para a seleção do tipo de esquema.

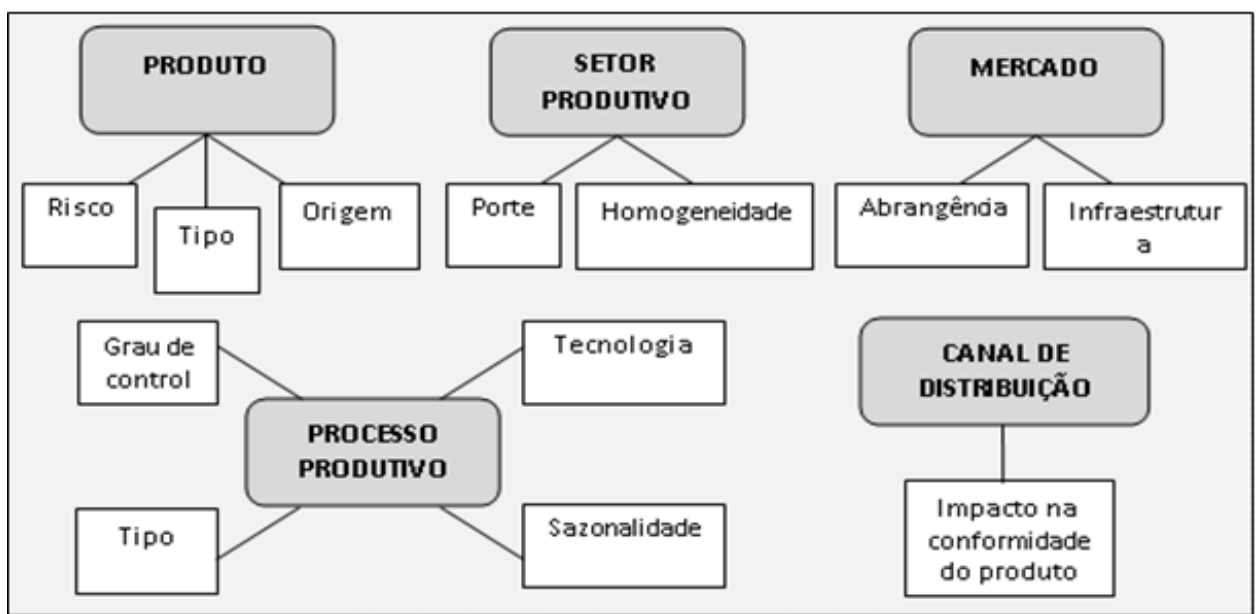

Fonte: Elaboração própria, 2016.

Adotando-se como referência o modelo CEN, foram atribuídos valores para as variáveis e as mesmas foram associadas às funções e dimensões para certificação de produtos. A função Supervisão e suas dimensões Existência, Atividades de Avaliação da Conformidade e Ponto de Coleta foram tidas como as mais relevantes para a seleção dos tipos de esquemas de certificação. $\mathrm{O}$

Quadro 3 resume as variáveis, os valores a elas atribuídos e a forma como deve influenciar a seleção do tipo de esquema de certificação.

Quadro 3: Variáveis, valores e influência na seleção dos tipos de certificação

\begin{tabular}{|c|c|c|}
\hline Variável & Valores atribuídos & Como influencia? \\
\hline Tipo de produto & $\begin{array}{c}\text { Produto; processo; } \\
\text { serviço. }\end{array}$ & Se processo ou serviço, o tipo de esquema deve ser o 6 . \\
\hline Risco do produto & $\begin{array}{l}\text { Baixo; moderado; } \\
\text { crítico. }\end{array}$ & Relaciona-se ao rigor do processo de avaliação da conformidade. \\
\hline $\begin{array}{l}\text { Origem do } \\
\text { produto }\end{array}$ & Nacional; importado & $\begin{array}{l}\text { Não determina a escolha do tipo de esquema de certificação, no } \\
\text { entanto, na existência de produtos importados, é sempre válido } \\
\text { considerar a adoção do tipo de esquema 1b como uma opção para } \\
\text { certificação. }\end{array}$ \\
\hline $\begin{array}{l}\text { Grau de controle } \\
\text { do processo }\end{array}$ & Baixo; médio; alto. & $\begin{array}{l}\text { Quanto mais controlado for o processo, menores são as chances de } \\
\text { fabricação de produtos fora das especificações e, assim, a } \\
\text { avaliação da conformidade pode ser menos rigorosa. }\end{array}$ \\
\hline $\begin{array}{l}\text { Tecnologia do } \\
\text { processo }\end{array}$ & $\begin{array}{l}\text { Baixa; média; alta } \\
\text { automação. }\end{array}$ & $\begin{array}{l}\text { Considerando ainda que um dos benefícios da automação é a } \\
\text { redução da variabilidade, a auditoria no SG e a avaliação da } \\
\text { processo produtivo podem ser dispensadas quão maior for a } \\
\text { automação, podendo restringir apenas em ensaios e/ou inspeções } \\
\text { do produto. Baixas tecnologias, por sua vez, podem até mesmo } \\
\text { requerer a avaliação lote a lote (1b). }\end{array}$ \\
\hline Tipo de processo & $\begin{array}{l}\text { Processos de baixo } \\
\quad \text { volume e alta } \\
\text { variedade; processos } \\
\text { de volume e } \\
\text { variedade medianos; }\end{array}$ & $\begin{array}{l}\text { Para os casos de processos de baixo volume e alta variedade, pode } \\
\text { ser aplicável o esquema de certificação de tipo, que não contempla } \\
\text { a etapa de Supervisão. Para os processos de volume e variedade } \\
\text { medianos e para os processos de alto volume e baixa variedade } \\
\text { empregam-se, em princípio, os tipos de esquemas que }\end{array}$ \\
\hline
\end{tabular}




\begin{tabular}{|c|c|c|}
\hline Variável & Valores atribuídos & Como influencia? \\
\hline & $\begin{array}{l}\text { processos de alto } \\
\text { volume e baixa } \\
\text { variedade. }\end{array}$ & $\begin{array}{l}\text { contemplem a etapa de Supervisão, de forma que produtos } \\
\text { fabricados regularmente sejam avaliados de forma periódica. }\end{array}$ \\
\hline $\begin{array}{l}\text { Sazonalidade da } \\
\text { produção }\end{array}$ & $\begin{array}{l}\text { Sim (exemplo: ovos } \\
\text { de páscoa e artigos de } \\
\text { carnaval); não. }\end{array}$ & $\begin{array}{l}\text { Caso a produção seja sazonal, recomenda-se a adoção de um tipo } \\
\text { de esquema de certificação que não inclua a função Supervisão } \\
\text { (esquemas tipo 1a ou 1b). Do contrário, é provável que as } \\
\text { atividades de avaliação da conformidade não possam ser } \\
\text { executadas em função da suspensão temporária da produção. }\end{array}$ \\
\hline $\begin{array}{l}\text { Porte do } \\
\text { fabricante }\end{array}$ & $\begin{array}{l}\text { Microempresa; } \\
\text { pequena empresa; } \\
\text { média/ grande } \\
\text { empresa }\end{array}$ & $\begin{array}{l}\text { O porte do fabricante é considerado um fator ponderador, não } \\
\text { estando associado à nenhuma função de avaliação da } \\
\text { conformidade ou dimensão. O tipo de esquema é selecionado em } \\
\text { função dos valores das diversas variáveis relacionadas ao produto, } \\
\text { ao processo produtivo, ao mercado e ao canal de distribuição. } \\
\text { Após, caso o fabricante solicitante da certificação se enquadre } \\
\text { como MPE, deve ser avaliada a conveniência de realizar a } \\
\text { substituição do tipo de esquema selecionado por outro menos } \\
\text { rigoroso. }\end{array}$ \\
\hline $\begin{array}{l}\text { Porte do } \\
\text { fabricante }\end{array}$ & $\begin{array}{l}\text { Microempresa; } \\
\text { pequena empresa; } \\
\text { média ou grande } \\
\text { empresa }\end{array}$ & $\begin{array}{l}\text { O porte do fabricante é considerado um fator ponderador, não } \\
\text { estando associado à nenhuma função de avaliação da } \\
\text { conformidade ou dimensão. O tipo de esquema é selecionado em } \\
\text { função dos valores das diversas variáveis relacionadas ao produto, } \\
\text { ao processo produtivo, ao mercado e ao canal de distribuição. } \\
\text { Após, caso o fabricante solicitante da certificação se enquadre } \\
\text { como MPE, deve ser avaliada a conveniência de realizar a } \\
\text { substituição do tipo de esquema selecionado por outro menos } \\
\text { rigoroso. }\end{array}$ \\
\hline $\begin{array}{l}\text { Homogeneidade } \\
\text { do setor }\end{array}$ & $\begin{array}{l}\text { Sim, para um setor } \\
\text { homogêneo; não, } \\
\text { para um setor } \\
\text { heterogêneo. }\end{array}$ & $\begin{array}{l}\text { A não homogeneidade do setor pode acarretar no emprego de mais } \\
\text { de um tipo de esquema de certificação de produto dentro de um } \\
\text { mesmo esquema de certificação para dar conta das diferentes } \\
\text { possibilidades de fornecimento de produtos, mas não influencia na } \\
\text { seleção do tipo de esquema de certificação. }\end{array}$ \\
\hline $\begin{array}{l}\text { Abrangência do } \\
\text { produto no } \\
\text { mercado }\end{array}$ & $\begin{array}{l}\text { Local; regional; } \\
\text { nacional. }\end{array}$ & $\begin{array}{l}\text { Afeta o ponto de coleta da amostra na etapa da Supervisão, } \\
\text { podendo ser na fábrica e/ou no mercado. Para um produto de } \\
\text { abrangência nacional, por exemplo, recomenda-se a utilização de } \\
\text { um tipo de esquema que inclua auditoria do SG, avaliação do } \\
\text { processo produtivo, além da realização de ensaios ou inspeções do } \\
\text { produto coletado ao menos na fábrica (esquema tipo 5). A } \\
\text { proposta visa a fortalecer ainda mais a avaliação pré-mercado de } \\
\text { forma a evitar a distribuição generalizada de produtos não } \\
\text { conformes para o mercado. }\end{array}$ \\
\hline $\begin{array}{l}\text { Infraestrutura } \\
\text { para fiscalização }\end{array}$ & Baixa; média; Alta. & $\begin{array}{l}\text { O acompanhamento no mercado deve estar em equilíbrio com o } \\
\text { processo de avaliação da conformidade e esse equilíbrio depende } \\
\text { da disponibilidade de recursos, da natureza do mercado que está } \\
\text { sendo regulado, da eficácia dos procedimentos de avaliação da } \\
\text { conformidade e da infraestrutura para fiscalização. Porém, não } \\
\text { influencia na seleção do tipo de esquema, sendo apenas fator } \\
\text { ponderador. }\end{array}$ \\
\hline $\begin{array}{l}\text { Impacto da } \\
\text { distribuição na } \\
\text { conformidade do } \\
\text { produto }\end{array}$ & Sim; não. & $\begin{array}{l}\text { Quando a distribuição impacta na conformidade do produto, deve } \\
\text { ser selecionado um tipo de esquema de certificação que inclua a } \\
\text { coleta de amostras no mercado para a realização de ensaios e/ou } \\
\text { inspeções. }\end{array}$ \\
\hline
\end{tabular}

Fonte: Elaboração própria, 2016.

A existência da Supervisão depende dos valores das variáveis tipo de produto, risco do produto, grau de controle do processo, tecnologia do processo, tipo de processo e sazonalidade da produção. As atividades de avaliação da conformidade conduzidas na etapa de Supervisão 
dependem dos valores assumidos pelas variáveis tipo de produto, risco do produto, grau de controle do processo, tecnologia do processo e abrangência do produto no mercado. O ponto de coleta das amostras para a realização de ensaios e/ou inspeções na etapa de Supervisão é influenciado pelas variáveis risco do produto, abrangência do produto no mercado e impacto do canal de distribuição na conformidade do produto.

As variáveis origem do produto, porte do fabricante, homogeneidade do setor e infraestrutura para fiscalização não foram diretamente associadas a nenhuma função de avaliação da conformidade, ainda que impactem na seleção dos tipos de esquemas de certificação. No que se refere à origem do produto, em havendo produtos importados no setor regulamentado, o esquema de certificação tipo 1 b deve ser considerado como uma das opções de escolha pelo solicitante da certificação. Para o caso de setores não homogêneos, cujos fabricantes apresentem diferentes características de processos produtivos, por exemplo, pode ser necessária a utilização de mais de um tipo de esquema de certificação. Na existência de micro e pequenas empresas (MPE), os procedimentos de certificação podem ser aliviados, mas a infraestrutura para fiscalização deve ser capaz de compensar no pós-mercado a diminuição de rigor da avaliação pré-mercado. Assim, o porte dos fabricantes e a infraestrutura para fiscalização são encarados como fatores ponderadores para o aumento ou diminuição de rigor dos procedimentos de certificação.

\subsection{SISTEMÁTICA PARA A SELEÇÃO DOS TIPOS DE ESQUEMA DE CERTIFICAÇÃO DE PRODUTOS}

A sistemática proposta é composta por três etapas. A Etapa 1, denominada "Caracterização do objeto da certificação", consiste no enquadramento do objeto em uma das três categorias atribuídas ao tipo de produto, quais sejam: produto, processo ou serviço. Caso o objeto seja um produto propriamente dito, segue-se para a Etapa 2. Caso o objeto seja um processo ou serviço, sua certificação deverá ser realizada de acordo com o esquema tipo 6, o que significa dizer que a certificação será baseada na realização de auditorias do SG e na avaliação do processo ou serviço. Caso o processo ou serviço resulte em algum elemento tangível, este elemento pode ser alvo de atividades de ensaio e/ou inspeção. Ou seja, o grau de intangibilidade do processo ou serviço é utilizado para definir se, além da auditoria do SG e da avaliação do processo/serviço, cabe a realização de outras atividades de determinação, como ensaio e/ou inspeção. 
A Etapa 2, "Caracterização do Cenário, é específica para os objetos enquadrados na Etapa 1 como "produtos". O objetivo desta etapa é selecionar, dentre os tipos de esquemas 1a, 1b, 2, 3, 4 e 5, o que melhor se adéqua à certificação de um produto específico, considerando o contexto particular em que está inserido. Deve ser considerada nesta etapa a homogeneidade do setor a partir da verificação da existência ou inexistência de diferenças entre os fabricantes. Caso o setor não seja homogêneo, deve-se proceder a análise de dois ou mais cenários, de forma a abranger todas as diferenças identificadas, com o propósito de definir se mais de um tipo de esquema de certificação deve ser indicado para adoção.

$\mathrm{Na}$ análise de cada cenário é feita a comparação dos valores de sete variáveis (sazonalidade da produção, tipo de processo, grau de controle do processo, tecnologia do processo, risco do produto, abrangência do produto no mercado e impacto na conformidade do produto) com as características dos tipos de esquemas de certificação. O Quadro 4 estabelece a relação os valores possíveis de cada variável e os tipos de esquema de certificação a serem escolhidos. Estas relações são resultado da correlação das variáveis com as funções e dimensões de cada tipo de esquema de certificação.

Quadro 4: Valores das variáveis para cada tipo de esquema de certificação.

\begin{tabular}{|c|c|c|c|c|c|c|}
\hline \multirow{2}{*}{ Variáveis } & \multicolumn{7}{|c|}{ Tipo de esquema de certificação } \\
\cline { 2 - 7 } & 1a & 1b & $\mathbf{2}$ & $\mathbf{3}$ & $\mathbf{4}$ & Não \\
\hline $\begin{array}{c}\text { Sazonalidade da } \\
\text { produção }\end{array}$ & $\begin{array}{c}\text { Baixo } \\
\text { volume e } \\
\text { alta } \\
\text { variedade }\end{array}$ & $\begin{array}{c}\text { Sim / Não } \\
\text { volume e } \\
\text { variedade } \\
\text { mediana } \\
\text { ou baixa }\end{array}$ & $\begin{array}{c}\text { Médio ou } \\
\text { alto } \\
\text { volume e } \\
\text { variedade } \\
\text { mediana } \\
\text { ou baixa }\end{array}$ & $\begin{array}{c}\text { Médio ou } \\
\text { alto } \\
\text { volume e } \\
\text { variedade } \\
\text { mediana } \\
\text { ou baixa }\end{array}$ & $\begin{array}{c}\text { Médio ou } \\
\text { alto } \\
\text { volume e } \\
\text { variedade } \\
\text { mediana } \\
\text { ou baixa }\end{array}$ & $\begin{array}{c}\text { Médio ou } \\
\text { alto } \\
\text { volume e } \\
\text { variedade } \\
\text { mediana ou } \\
\text { baixa }\end{array}$ \\
\hline $\begin{array}{c}\text { Grau de controle do } \\
\text { processo }\end{array}$ & --- & Baixo & Alto & Médio & Médio & Baixo \\
\hline Tecnologia do processo & --- & Baixa & Alta & Média & Média & Baixa \\
\hline $\begin{array}{c}\text { Risco do produto } \\
\text { Baixo } \\
\text { (P baixa e } \\
\text { C baixa) }\end{array}$ & $\begin{array}{c}\text { Crítico } \\
\text { alta e C }\end{array}$ & $\begin{array}{c}\text { Baixo } \\
\text { (P média e } \\
\text { C baixa) }\end{array}$ & $\begin{array}{c}\text { Baixo } \\
\text { (P baixa e } \\
\text { C média) }\end{array}$ & $\begin{array}{c}\text { Moderado } \\
\text { (P média } \\
\text { ou C } \\
\text { média) }\end{array}$ \\
\hline $\begin{array}{c}\text { Abrangência do produto } \\
\text { no mercado }\end{array}$ & --- & --- & Local & Regional & Regional & Nacional \\
\hline $\begin{array}{c}\text { Impacto (do canal) na } \\
\text { conformidade do produto }\end{array}$ & --- & --- & Sim & Não & Sim / Não & Sim / Não \\
\hline
\end{tabular}

Fonte: Elaboração própria, 2016

É possível que os valores das variáveis não resultem em uma combinação perfeita com um único tipo de esquema de certificação. Dessa forma, uma das possibilidades de decisão é eleger o tipo de esquema que apresentar o maior número de correlações positivas. Em caso de um "quase empate" entre dois tipos de esquemas de certificação, uma alternativa pode ser 
selecionar o tipo de esquema que apresente correlação com variáveis consideradas pelo regulamentador como as mais críticas (por exemplo, risco do produto), ao invés de simplesmente adotar o tipo de esquema com o maior número de correlações positivas. Outra possibilidade de ação é conduzir uma Análise de Impacto Regulatório (AIR), considerando as mais de uma opção de tipos de esquemas para cada cenário, de forma a selecionar o tipo de esquema com menor impacto.

Ainda na Etapa 2, outra variável que deve ser considerada é a origem do produto. Caso haja produtos importados, o esquema de certificação de lote (tipo 1b) deve ser considerado como uma das opções de uso.

Finalmente, chega-se na Etapa 3, "Ponderação". Somente duas variáveis são consideradas como fatores de ponderação para a escolha do tipo de esquema para a certificação de um produto: o porte do fabricante e a infraestrutura para fiscalização. Na existência de MPE, é recomendável que os procedimentos de certificação sejam aliviados. Assim, o regulamentador deve avaliar se é apropriado adotar um tipo de esquema de certificação menos rigoroso do que o selecionado na Etapa 2. É essencial que essa decisão também esteja fundamentada pela análise do nível de infraestrutura para fiscalização, de forma a manter o desejável equilíbrio entre controle pré-mercado e pós-mercado. Não obstante o risco de o produto ter sido considerado na etapa anterior, ele deve ser novamente analisado para a tomada de decisão.

Alguns critérios balizadores para essa tomada de decisão devem ser seguidos. Para a substituição de um tipo de esquema que contemple a etapa de Supervisão por um tipo de esquema que não abranja essa função (esquema tipo 1a), recomenda-se a associação de um esquema de DF à certificação de "tipo". Nesse caso, é possível estender o uso da marca de conformidade para os produtos fabricados após a certificação de tipo (1a), com base na própria declaração do fornecedor. Para a substituição de um tipo de esquema que contemple a auditoria do SG e/ou a avaliação do processo produtivo por um tipo de esquema que compreenda apenas ensaios e/ou inspeções (esquema tipo 2), convém que seja reduzido o intervalo entre as Supervisões. Já para produtos de risco moderado ou crítico, a substituição de um tipo de esquema por outro menos rigoroso requer o fortalecimento das ações de fiscalização.

Para o caso específico de processos e serviços, a "Ponderação" pode impactar especialmente a definição da frequência da Supervisão. A ponderação deve considerar o risco do processo ou serviço, o porte do fornecedor e a infraestrutura para fiscalização. Os seguintes critérios balizadores devem ser assumidos. Quando o risco do processo for moderado ou crítico, o fornecedor não se enquadrar nas condições de MPE e a infraestrutura para fiscalização for baixa, recomenda-se intervalos curtos entre as Supervisões. Já quando o risco do processo for 
moderado ou crítico, o fornecedor se enquadrar nas condições de MPE e a infraestrutura para fiscalização for média ou alta, pode-se optar por exigir apenas a avaliação do processo ou serviço, sem exigir a realização de auditorias periódicas.

\section{CONSIDERAÇÕES FINAIS}

Os resultados obtidos podem ser utilizados por outros agentes regulamentadores de diferentes órgãos além do Inmetro que decidam fazer uso da certificação no apoio às suas atividades de regulamentação. Até mesmo os OCP, no estabelecimento de seus esquemas próprios de certificação de produtos, processos e serviços, poderão se apoiar na sistemática com poucos ajustes a serem feitos. A aplicação da sistemática proposta proporciona maior sistematização no estabelecimento de requisitos de avaliação da conformidade para a certificação de produtos, processos e serviços, conferindo melhoria da qualidade regulatória. Adicionalmente, confere maior transparência à atividade de regulamentação técnica, promovendo também participação mais efetiva das partes interessadas e impactadas. A aplicação, pelo Inmetro uo por outro regulamentador, da sistemática guarda alguns pontos de atenção, descritos a seguir.

Para setores com moderado ou alto grau de competição, que abrangem um grande número de fabricantes ou importadores, é possível que sejam identificados distintos cenários, com empresas que se diferenciem nos seus processos produtivos e na forma como os seus produtos são distribuídos para o mercado. É fundamental, portanto, que seja feito um mapeamento robusto do setor regulamentado antes de iniciar o desenvolvimento de um programa de avaliação da conformidade. Sugere-se que a análise de cenários e a definição dos valores de cada variável seja feita na fase de Análise de Impacto Regulatório (AIR), etapa anterior ao processo de desenvolvimento do regulamento. Essa sugestão vai ao encontro das diretrizes da ISO, que preconiza o uso da AIR na análise das opções de uso da avaliação da conformidade na atividade de regulamentação.

A aplicação do método requer também que, para o caso em que se disponibiliza mais de um tipo de esquema de certificação, sejam declarados os critérios de acesso a cada um deles. Resgata-se aqui a diretriz do Conmetro para que os regulamentadores definam claramente o grau de liberdade de escolha entre os procedimentos de avaliação da conformidade nos casos em que mais de um procedimento é utilizado em um regulamento. Dessa forma, diante de cenários diversos, cabe ao regulamentador definir, de forma objetiva, as condições que devem ser atendidas pelo solicitante da certificação para acessar um ou outro tipo de esquema de 
certificação que componha o esquema de certificação de um determinado objeto, cabendo aos Organismos de Certificação de Produtos (OCP), no processo de certificação, verificar se as condições de acesso ao tipo de esquema são de fato atendidas.

Não obstante os desafios para sua aplicação, a aplicação da sistemática para seleção de tipos de esquemas de certificação será útil ao Inmetro e poderá contribuir para a melhoria da qualidade do seu processo regulatório. É com muita satisfação que se anuncia que os critérios propostos nesta pesquisa já foram utilizados pelo Inmetro na Análise de Impacto Regulatório e estão sendo internalizados na norma interna de desenvolvimento e aperfeiçoamento de medidas regulatórias. 


\section{REFERENCIAS}

ASSOCIAÇÃO BRASILEIRA DE NORMAS TÉCNICAS. NBR ISO/IEC 17067: Avaliação da conformidade - Fundamentos para certificação de produtos e diretrizes de esquemas para certificação de produtos. Rio de Janeiro: ABNT, 2015.

ASSOCIAÇÃO BRASILEIRA DE NORMAS TÉCNICAS. NBR ISO/IEC 17065: Avaliação da conformidade - Requisitos para organismos de certificação de produtos, processos e serviços. Rio de Janeiro: ABNT, 2013.

ASSOCIAÇÃO BRASILEIRA DE NORMAS TÉCNICAS. ISO/IEC GUIA 67: Avaliação de conformidade - Fundamentos de certificação de produtos. Rio de Janeiro: ABNT, 2005a.

ASSOCIAÇÃO BRASILEIRA DE NORMAS TÉCNICAS. NBR ISO/IEC 17000: Avaliação da conformidade - Vocabulário e princípios gerais. Rio de Janeiro: ABNT, 2005b.

ASSOCIAÇÃO NACIONAL DE PÓS-GRADUAÇÃO E PESQUISA EM

ADMINISTRAÇÃO, 30., 2006, Salvador. Disponível em:

http://www.anpad.org.br/enanpad/2006/dwn/enanpad2006-adid-0069.pdf. Acesso em: 10 fev. 2015 .

ASIA-PACIFIC ECONOMIC COOPERATION. Good regulatory practices for conformity assessment in APEC member economies. APEC, 2013. Disponível em:

http://publications.apec.org/publication-detail.php?pub_id=1473. Acesso em: 06 out. 2015.

ASIA-PACIFIC ECONOMIC COOPERATION. Good practice guide on regulatory reform. APEC, 2010. Disponível em:

http://publications.apec.org/googlesearch.php?cx=012092251120059988616\%3Atcuzscmwdt a\&cof=FORID\%3A11\&q=regulation\&sa=. Acesso em: 23 set. 2015.

BARBULIO FILHO, Arnaldo. Aplicação da ISO 17067: Workshop. 2012.

BRASIL. Lei $\mathbf{n}^{\mathbf{0}} \mathbf{1 2 . 5 4 5}$, de 14 de dezembro de 2011. Dispõe sobre o fundo de financiamento à exportação (FFEX), altera o art. $1^{\circ}$ da lei $n^{\circ} 12.096$, de 24 de novembro de 2009 , e as leis $n^{\circ}$ 10.683, de 28 de maio de 2003, 11.529, de 22 de outubro de 2007, 5.966, de 11 de dezembro de 1973, e 9.933, de 20 de dezembro de 1999; e dá outras providências. 2011. Disponível em: http://www.inmetro.gov.br/legislacao. Acesso em: 16 jun. 2015.

BRASIL. Lei no 9.933, de 20 de dezembro de 1999. Dispõe sobre as competências do Conmetro e do Inmetro, institui a taxa de serviços metrológicos, e dá outras providências. 1999. Disponível em: http://www.inmetro.gov.br/legislacao. Acesso em: 16 jun. 2015.

CHAMUSCA, Roberta F.; SAMPAIO, Cristiane M. S.; SILVA, Danielle A. V. S. Certificação como apoio à regulamentação de produtos, processos e serviços: o caso do Inmetro. In: CONGRESSO BRASILEIRO DE REGULAÇÃ̃O, 9., 2015, Brasília.

COMISSÃO EUROPEIA. Guia azul relativo à aplicação da legislação da UE em matéria de produtos. Ed. 1.1. CE, 2015. Disponível em: http://ec.europa.eu/DocsRoom/documents/11502. Acesso em: 18 set. 2015. 
COMITÊ EUROPEU DE NORMALIZAÇÃO. CEN/BT WG 185 Project Team Final draft report. Genova: AENOR, 2018. (Project team final report for approval: European accessibility requirements for public procurement of products and services in the ICT domain - European Commission Mandate M 376, Phase 1. 2008). Disponível em: http://www.econformance.eu/documents/BTWG185_N30_PT_Final_Report_for_Approval.p df. Acesso em: 29 jan. 2016.

CONSELHO NACIONAL DE METROLOGIA, NORMALIZAÇÃO E QUALIDADE INDUSTRIAL Guia de boas práticas de regulamentação. Brasília: CONMETRO, 2007. Disponível em: http://www.inmetro.gov.br/qualidade/guiaRegulamentacao.asp. Acesso em: 21 jan. 2015.

DCMAS. Building corresponding technical infrastructures to support sustainable development and trade in developing countries and countries in transition: Background paper. 2010. Disponível em: http://www.dcmas.net. Acesso em: 21 jan. 2015.

FOSSA, Alberto J. Contribuições para a modelagem do sistema de avaliação da conformidade na construção da infraestrutura predial para distribuição e uso residencial de gases combustíveis. 2012. 275 f. Tese (Doutorado em Energia) - Universidade de São Paulo, São Paulo, 2014.

GIL, Antonio C. Como elaborar projetos de pesquisa. 5. ed. São Paulo: Atlas, 2010.

INSTITUTO NACIONAL DE METROLOGIA, QUALIDADE E TECNOLOGIA. Avaliação da conformidade. 6. ed. Rio de Janeiro: INMETRO, 2015a. Disponível em: http://www.inmetro.gov.br/inovacao/publicacoes/acpq.pdf. Acesso em: 29 jan. 2016.

INTERNATIONAL ORGANIZATION FOR STANDARDIZATION. Using ISO/CASCO standards in regulations. Geneva: ISO, [201-]. Disponível em: https://www.iso.org/sites/cascoregulators/documents/casco-regulators-fulltext.pdf. Acesso em: 29 jun. 2015.

INTERNATIONAL ORGANIZATION FOR STANDARDIZATION. Certification and related activities: assessment and verification of conformity to standards and technical specifications. Geneva: ISO, 1992.

INTERNATIONAL ORGANIZATION FOR STANDARDIZATION. Certificación: princípios y práctica. Madrid: IRANOR, 1982.

INTERNATIONAL ORGANIZATION FOR STANDARDIZATION; UNITED NATIONS INDUSTRIAL DEVELOPMENT ORGANIZATION. Building trust: the conformity assessment toolbox. Genebra: ISO; Viena: UNIDO, 2010. Disponível em: http://www.iso.org/iso/home/store/publication_item.htm?pid=PUB100230. Acesso em: 17 maio 2014.

LODGE, Martin; WEGRICH, Kai. O enraizamento da regulação de qualidade: fazer as perguntas difíceis é a resposta. In: PROENÇA, Jadir P.; COSTA, Patrícia V.;

MONTAGNER, Paula (org.). Desafios da regulação no Brasil. Brasília: ENAP, 2009. p. 17-37. Disponível em: http://www.regulacao.gov.br/acompanhe-o-pro-reg/livros/desafiosda-regulacao-no-brasil.pdf/view. Acesso em: 26 set. 2015. 
RUSSO, Giuseppe M.; MACEDO-SOARES, T. Diana L. A.; VILLAS, Marcos V. Importância da hierarquização das revistas científicas: resultados de uma investigação empírica no Brasil e proposta de um método de pesquisa bibliográfica. In: ENCONTRO DA

UNIÃO EUROPEIA (UE). Decisão no 768/2008/CE, de 9 de julho de 2008, relativa a um quadro comum para a comercialização de produtos. Disponível em: http://eurlex.europa.eu/LexUriServ/LexUriServ.do?uri=OJ:L:2008:218:0082:0128:pt:PDF. Acesso em: 18 set. 2015.

UNITED NATIONS ECONOMIC COMMISSION FOR EUROPE (UNECE). Risk management in regulatory frameworks: towards a better management of risks. United Nations: New York and Geneva, 2012. Disponível em:

http://www.unece.org/trade/wp6/welcome.html. Acesso em: 21 jan. 2015.

UNITED STATE. European Commission. New legislative framework. Belgica: EU, [201-]. Disponível em: http://ec.europa.eu/growth/single-market/goods/new-legislative-framework. Acesso em: 30 out. 2015. 